\title{
Efficacy of Neoadjuvant Chemotherapy DOX and XELOX Regimens for Patients with Resectable Gastric or Gastroesophageal Junction Adenocarcinoma
}

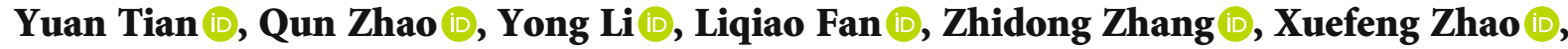 \\ Bibo Tan $₫$, Dong Wang $₫$, and Peigang Yang $₫$ \\ Department of General Surgery, The Fourth Affiliated Hospital, Hebei Medical University, No.12, Jian-Kang Road, \\ Shijiazhuang 050019, China \\ Correspondence should be addressed to Qun Zhao; zhaoqun@hebmu.edu.cn
}

Received 6 January 2021; Revised 29 June 2021; Accepted 1 July 2021; Published 23 July 2021

Academic Editor: Nicola Silvestris

Copyright (c) 2021 Yuan Tian et al. This is an open access article distributed under the Creative Commons Attribution License, which permits unrestricted use, distribution, and reproduction in any medium, provided the original work is properly cited.

Purpose. This paper is aimed at comparing the short-term efficacy of the combination of docetaxel, oxaliplatin, and capecitabine (DOX) with the combination of oxaliplatin and capecitabine (XELOX) as neoadjuvant chemotherapy regimens for the treatment of patients with resectable gastric or gastroesophageal junction adenocarcinoma. Methods. A total of 300 patients aged 20-60 years with resectable gastric or gastroesophageal junction adenocarcinoma who were evaluated with cT3/4Nany were randomly assigned into 3 groups: DOX group ( $n=100$, treated with neoadjuvant DOX plus adjuvant XELOX), XELOX group ( $n=100$, treated with perioperative XELOX), and surgery group $(n=100$, treated with adjuvant XELOX). Results. A total of 93 , 92, and 95 patients were enrolled in the DOX, XELOX, and surgery groups, respectively. The pathological complete response (pCR) rate was $16.1 \%$ in the DOX group and $4.3 \%$ in the XELOX group $(P=0.008)$. There were $56(61.3 \%)$ patients in the DOX group who presented with surgical complications, $22(23.9 \%)$ patients in the XELOX group, and $37(38.9 \%)$ patients in the surgery group. The most common grade $3-4$ adverse events in these three groups were neutropenia $(32.3 \%, 30.4 \%$, and $21.1 \%)$, leucopenia $(21.5 \%, 22.8 \%$, and $15.8 \%)$, nausea $(15.1 \%, 16.3 \%$, and $12.6 \%)$, and fatigue $(10.8 \%, 7.6 \%$, and $8.4 \%)$. Conclusions. Neoadjuvant DOX is an effective and feasible regimen and might represent an option for young and middle-aged patients with locally advanced, resectable gastric or gastroesophageal junction adenocarcinoma.

\section{Introduction}

More than $50 \%$ of gastric cancer (GC) patients are neglected and diagnosed with a locally advanced stage as there is a lack of specific screening programs. Moreover, even in the cases of curative resection, the prognosis of patients with positive lymph node metastases remains poor, with five-year survival rates of $20-30 \%$ [1]. The effect of neoadjuvant chemotherapy for advanced GC has been accepted by more and more clinicians. According to NCCN guidelines [2], the FLOT regimen (docetaxel, oxaliplatin, fluorouracil, and leucovorin) was recommended. However, in the prospective clinical trials of the FLOT regimen [3-6], rare Asian patients are included, and the efficacy and tolerance of the regimen to Chinese GC patients remain unclear. Our previous clinical trial (NCT01516944) has shown the efficacy and safety of the XELOX regimen (oxaliplatin and capecitabine) and SOX regimen (tegafur, gimeracil, and oteracil potassium (S-1) plus oxaliplatin) as neoadjuvant chemotherapy $[7,8]$. Docetaxel is the key treatment for metastatic GC drugs. Based on previous results [8], whether the docetaxel-based triplet chemotherapy DOX regimen can improve the pathological complete response (pCR) rate, prolong the survival, and obtain better tolerance or not needs to be confirmed. Considering potential better efficacy and more toxicity of triplet chemotherapy compared with doublet chemotherapy, middle- 


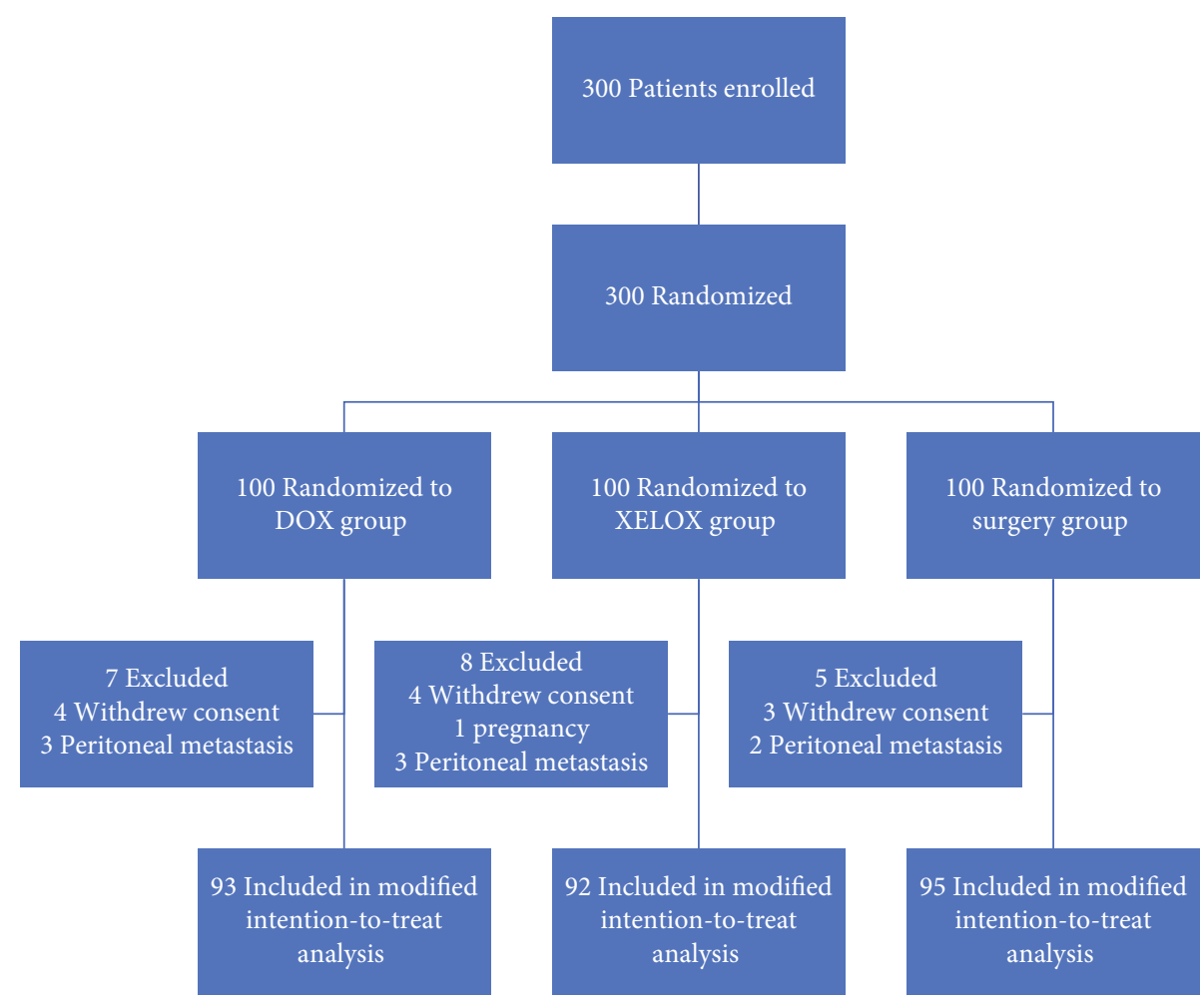

FIGURE 1: Trial profile. DOX = capecitabine, oxaliplatin, and docetaxel; XELOX = capecitabine and oxaliplatin.

aged and young patients were enrolled in this study. Pathologic tumor response (pCR) has been shown as a controversial but promising survival marker in GC. Therefore, this paper mainly focuses on the short-term efficacy pCR rate.

\section{Materials and Methods}

2.1. Study Design and Participants. This study was an investigator-initiated multicenter, randomized, open-label, and controlled trial, and it was conducted in accordance with the principles of the Helsinki declaration. This trial was approved by the Medical Ethics Committee of the Fourth Hospital of Hebei Medical University (No. 2014020) and registered on ClinicalTrials.gov (NCT02555358, 21/09/2015). All 300 patients were centrally randomized $1: 1: 1$ to neoadjuvant DOX plus adjuvant XELOX or perioperative XELOX or adjuvant XELOX by using an interactive web-response system (IWRS). Patients were enrolled by authorized individuals who requested randomization with an IWRS integrated into the electronic case report forms (eCRF). Assignment to trial groups was completed on the server of the independent data management providers (Bioknow, Beijing, China) via a validated assignment program, which underlies strict access control. The randomization system assigned each patient a unique identification number and sent the researchers a message containing the results of the assignment.

Patients were enrolled according to the following inclusion criteria: histologically or cytologically proved operable advanced gastric adenocarcinoma, being identified as a potentially resectable patient (cT3-4,Nany,M0) by a multidisciplinary consultation, Karnofsky Performance Scale
(KPS) score $>80$, Eastern Cooperative Oncology Group (ECOG) performance status of $0-1$, expected survival $>6$ months, age 20-60 years, voluntary participation in the study and signing the informed consent forms, and adequate major organ functions (neutrophil count $\geq 1.5 \times 10^{9} / \mathrm{L}$, platelet count $\geq 100 \times 10^{9} / \mathrm{L}$, hemoglobin $\geq 90 \mathrm{~g} / \mathrm{L}$, liver function $<$ 1.5 times of the upper limit of normal, serum bilirubin $\leq$ $1.0 \times \mathrm{UNL}$, serum creatinine $<1.5 \times \mathrm{UNL}$, and PTINR/PTT $<1.7$ times of the upper limit of normal).

2.2. Procedures. Neoadjuvant DOX or XELOX was administered for 4 cycles followed by 4 cycles of postoperative XELOX for the DOX or XELOX group. Eight XELOX postoperative cycles were administered for the surgery group. DOX consisted of docetaxel $60 \mathrm{mg} / \mathrm{m}^{2}$ intravenously on day 1 , oxaliplatin $130 \mathrm{mg} / \mathrm{m}^{2}$ intravenously on day 1 , and capecitabine $1000 \mathrm{mg} / \mathrm{m}^{2}$ p.o. (two doses of $500 \mathrm{mg} / \mathrm{m}^{2}$ per day) on day 1 to 14 , every 3-week cycle. XELOX included oxaliplatin $130 \mathrm{mg} / \mathrm{m}^{2}$ intravenously on day 1 and capecitabine $1000 \mathrm{mg} / \mathrm{m}^{2}$ given orally $\left(500 \mathrm{mg} / \mathrm{m}^{2}\right.$ twice a day) on day 1 to 14 , every 3 -week cycle. In patients with febrile neutropenia (despite the use of granulocyte colony-stimulating factor (GCSF)), thrombocytopenia that causes bleeding, or any other hematological dose-limiting toxicities, dosing of docetaxel and oxaliplatin reduced to $75 \%$. For grade $>2$ nonhematological toxicities, the dose of all drugs was reduced to $75 \%$; for grade 2 , the dose was reduced to $50 \%$ if toxicity recurred after the first dose of reduction. Treatment continued until intolerable toxicity, disease progression or death, withdrawal of consent, or investigator's decision. Prior to surgical treatment, CT or MRI and endoscopy were performed to rule 
TABLE 1: General clinical characteristics of patients in the three groups.

\begin{tabular}{lccccc}
\hline & $\begin{array}{c}\text { DOX } \\
N=100\end{array}$ & $\begin{array}{c}\text { XELOX } \\
N=100\end{array}$ & $\begin{array}{c}\text { Surgery } \\
N=100\end{array}$ & $\chi^{2}$ & $\begin{array}{c}P \\
\text { value }\end{array}$ \\
\hline Age (year) & & & & 1.520 & 0.468 \\
$<45$ & 15 & 17 & 11 & & \\
$45-60$ & 85 & 83 & 89 & & \\
\hline Sex & & & & 0.694 & 0.707 \\
$\mathrm{M}$ & 73 & 69 & 74 & & \\
$\mathrm{~F}$ & 27 & 31 & 26 & & \\
\hline ECOG & & & & 0.770 & 0.681 \\
0 & 38 & 42 & 44 & & \\
1 & 62 & 58 & 56 & & \\
\hline Tumor center & & & & 5.768 & 0.450 \\
EGJ & 33 & 38 & 28 & & \\
Gastric body & 11 & 14 & 10 & & \\
Gastric antrum & 52 & 42 & 53 & & \\
Other & 4 & 6 & 9 & & \\
\hline
\end{tabular}

Pathological type

$2.362 \quad 0.669$

High and medium

differentiation

Poor differentiation

Very low

differentiation

$1.510 \quad 0.470$

\begin{tabular}{llllll}
\hline cT stage & & & & 1.510 & 0.470 \\
cT3 & 28 & 31 & 36 & & \\
cT4 & 72 & 69 & 64 & & \\
\hline
\end{tabular}

\begin{tabular}{lccccc}
\hline $\mathrm{cN}$ stage & & & & 7.382 & 0.287 \\
$\mathrm{cN} 0$ & 10 & 16 & 12 & & \\
$\mathrm{cN} 1$ & 35 & 32 & 41 & & \\
$\mathrm{cN} 2$ & 41 & 46 & 35 & & \\
$\mathrm{cN} 3$ & 14 & 6 & 12 & & \\
\hline
\end{tabular}

Borrmann type

$9.769 \quad 0.135$

\begin{tabular}{lccc} 
I & 3 & 0 & 0 \\
II & 35 & 39 & 31 \\
III & 55 & 56 & 66 \\
IV & 7 & 5 & 3 \\
\hline
\end{tabular}

Very low differentiation: signet ring cell carcinoma, mucous adenocarcinoma, and anaplastic carcinoma.

out disease progression or distant metastasis and then every 2 cycles until disease progression, recurrence, or death. The tumor volume reduction rate of $12.5 \%$ was measured by CT as an effective threshold for evaluating neoadjuvant therapy. Surgery was scheduled 4 to 6 weeks after the completion of the last cycle of neoadjuvant chemotherapy. Surgeons had to be specialized abdominal surgeons. The tumor regression grade was quantified using the NCCN Clinical Practice Guidelines in Oncology (2014.v1). TRG0 was defined as complete response without viable cancer cells, TRG1 was near complete response with single cells or rare small groups of cancer cells, TRG2 was a partial response with residual cancer cells with evident tumor regression but more than single cells or rare small groups of cancer cells, and TRG3 was poor or no response with extensive residual cancer without evident tumor regression.

Pathological staging, including depth of tumor invasion (T), lymph node involvement $(\mathrm{N})$, and resection status (RX, $\mathrm{R} 0$, or R1), was judged by the local pathologist according to the $7^{\text {th }}$ edition of the TNM American Joint Committee on Cancer (AJCC) classification. Adverse events were assessed as per National Cancer Institute Common Terminology Criteria for Adverse Events (NCI-CTC, version 3.0).

2.3. Statistical Analysis. The sample size of this study was calculated based on the hypothesis that $5 \%$ of pCR was achieved in the XELOX group and 15\% in the DOX group. A total of 300 patients were calculated to provide $80 \%$ of the power to detect this improvement in pCR (one-sided significance level of $P<0.05$; Fisher's exact test), including $15 \%$ dropout approximately.

The analysis was conducted in the intent-to-treat (ITT) population and per-protocol (PP). Data were analyzed with SAS (version 9.3). Two-sided $P$ values were calculated using Fisher's exact test, unless otherwise indicated.

\section{Results}

Between September 2014 and June 2018, a total of 300 patients were screened (Figure 1). There were 93, 92, and 95 patients enrolled in the DOX group, the XELOX group, and the surgery group, respectively.

The baseline characteristics of the population are shown in Table 1. Patients in the DOX group included 63 males and 37 females with a median age of 52 years (range 3360). Seventy-one males and 29 females with a median age of 54 years were included in the XELOX group. Sixty-nine males and 31 females with a median age of 52.5 years consisted the surgery group.

The average number of preoperative cycles was 3.2 in the DOX group and 3.9 in the XELOX group. There were 79 $(84.9 \%)$ patients in the DOX group, $74(80.4 \%)$ patients in the XELOX group, and $83(87.4 \%)$ patients in the surgery group who received postoperative chemotherapy. A total of $31(33.3 \%)$ patients in the DOX group completed the study according to the protocol (8 cycles), $37(40.2 \%)$ patients in the XELOX group (8 cycles), and 27 (28.4\%) patients in the surgery group. In addition, there were $85(91.4 \%)$ patients who underwent surgery in the DOX group, 89 (96.7\%) patients in the XELOX group, and 95 (100\%) patients in the surgery group. Reasons for not proceeding to surgery were a progression of the disease, death, or metastatic disease detected after randomization, irresistibility detected during surgery, and patient request (Figure 1).

The tumor volumes before and after neoadjuvant chemotherapy in the DOX group were $52.13 \pm 25.63 \mathrm{~mm}^{3}$ and $42.55 \pm 19.31 \mathrm{~mm}^{3}$, respectively. The effective rate was $44.1 \%$ (14/93) of tumor volume reduction on CT. The tumor volumes before and after neoadjuvant chemotherapy in the XELOX regimen were $48.34 \pm 21.56 \mathrm{~mm}^{3}$ and $37.32 \pm 28.83$ 
TABLE 2: Serious adverse events with perioperative morbidity.

\begin{tabular}{lccccc}
\hline & DOX $(n=93)$ & XELOX $(n=92)$ & Surgery $(n=95)$ & $\chi^{2}$ & $P$ value \\
\hline $\begin{array}{l}\text { Patients with at least one serious adverse } \\
\text { event involving a perioperative morbidity }\end{array}$ & 31 & 22 & 20 & 4.008 & 0.135 \\
Surgical complication & & & & & \\
$\quad$ Pneumonia & 8 & 7 & 7 & 0.11 & 0.946 \\
Pleural complication & 15 & 5 & 11 & 5.41 & 0.067 \\
Chyle leakage & 1 & 0 & 0 & 2.018 & 0.365 \\
Seroperitoneum & 1 & 1 & 1 & 0.001 & 1.000 \\
Anastomotic fistula & 1 & 1 & 0 & 1.035 & 0.596 \\
Intestinal occlusion & 0 & 0 & 1 & 1.954 & 0.376 \\
\hline
\end{tabular}

TABLe 3: Adverse events in 3 groups.

\begin{tabular}{|c|c|c|c|c|c|c|c|c|c|}
\hline & \multicolumn{3}{|c|}{ DOX 93} & \multicolumn{3}{|c|}{ XELOX 92} & \multicolumn{3}{|c|}{ Surgery 95} \\
\hline & Grades 1-2 & Grade 3 & Grade 4 & Grades 1-2 & Grade 3 & Grade 4 & Grades 1-2 & Grade 3 & Grade 4 \\
\hline \multicolumn{10}{|c|}{ Gastrointestinal disorders } \\
\hline Nausea & 41 & 14 & 0 & 36 & 15 & 0 & 38 & 12 & 0 \\
\hline Vomiting & 20 & 4 & 0 & 10 & 2 & 0 & 12 & 0 & 0 \\
\hline Diarrhea & 34 & 6 & 0 & 26 & 2 & 0 & 16 & 6 & 0 \\
\hline Constipation & 8 & 0 & 0 & 8 & 0 & 0 & 4 & 0 & 0 \\
\hline Decreased appetite & 46 & 8 & 0 & 38 & 8 & 0 & 40 & 6 & 0 \\
\hline \multicolumn{10}{|l|}{ Dysphagia } \\
\hline \multicolumn{10}{|c|}{ Blood and lymphatic system disorders } \\
\hline Anemia & 44 & 6 & 2 & 44 & 2 & 0 & 38 & 4 & 0 \\
\hline Leucopenia & 54 & 16 & 4 & 50 & 17 & 4 & 46 & 15 & 0 \\
\hline Neutropenia & 59 & 24 & 6 & 48 & 26 & 2 & 40 & 18 & 2 \\
\hline Thrombocytopenia & 22 & 4 & 2 & 16 & 0 & 0 & 18 & 0 & 0 \\
\hline Febrile neutropenia & NA & 2 & 0 & NA & 0 & 0 & NA & 0 & 0 \\
\hline \multicolumn{10}{|c|}{ General and other disorders } \\
\hline Neurotoxic effects & 14 & 0 & 0 & 12 & 0 & 0 & 8 & 0 & 0 \\
\hline Fatigue & 46 & 10 & 0 & 38 & 7 & 0 & 34 & 8 & 0 \\
\hline Alopecia & 58 & NA & NA & 0 & NA & NA & 0 & NA & NA \\
\hline Weight decreased & 34 & 4 & 0 & 38 & 0 & 0 & 28 & 0 & 0 \\
\hline Skin effects & 8 & 0 & 0 & 4 & 0 & 0 & 2 & 0 & 0 \\
\hline \multicolumn{10}{|l|}{ Laboratory } \\
\hline ALT elevation & 14 & 2 & 0 & 10 & 0 & 0 & 8 & 0 & 0 \\
\hline GOT elevation & 10 & 2 & 0 & 12 & 0 & 0 & 10 & 0 & 0 \\
\hline
\end{tabular}

$\mathrm{mm}^{3}$, respectively. The effective rate was $26.1 \%$ (24/92) tumor volume reduction on $\mathrm{CT}$.

All patients underwent laparoscopic exploration before the radical resection. The $\mathrm{R} 0$ resection rates in the DOX group, the XELOX group, and the surgery group were $97.6 \%(83 / 85)$, $95.5 \%(85 / 89)$, and $94.7 \%$ (90/95), respectively. In the DOX group, 2 patients underwent gastrojejunostomy. In the XELOX group, 2 patients underwent laparoscopy and 2 underwent palliative resection. In the surgery group, 5 patients underwent palliative resection. A significantly higher proportion of patients achieved a pathological complete regression in the DOX group than in the XELOX group $(16.1 \%(15 / 93)$ in the DOX group vs. $4.3 \%$ (4/92) in the XELOX group; $P=$
0.008). The proportion of patients who achieved complete and subtotal regression was also higher with DOX $(41.9 \%$ (39/93)) than with XELOX (19.6\% (17/92); $P=0.04)$. The numbers of resected lymph nodes in the DOX group, the XELOX group, and the surgery group were $36.1 \pm 8.4,35.8$ \pm 9.2 , and $39.8 \pm 9.7$, respectively, and this difference was not statistically significant $(t=1.725, P=0.104)$. The proportion of patients with lymph node metastasis was 6.5\% $(218 / 3362)$ in the DOX group, $8 \%(252 / 3150)$ in the XELOX group, and $20.3 \%(767 / 3785)$ in the surgery group, and this difference was statistically significant $(t=108.065, P=0.001)$.

There were $33.3 \%$ (31/93) patients in the DOX group presented with surgical complications, while $23.9 \%(22 / 92)$ 
in the XELOX group and 21.1\% (20/95) in the surgery group (Table 2). Grade 3-4 nonsurgical adverse events were reported in 38 (40.9\%) of 93 patients in the DOX group, 30 (32.6\%) of 92 patients in the XELOX group, and 22 (23.2\%) of 95 patients in the surgery group. The most frequent grade 3-4 adverse events in three groups were neutropenia (32.3\% (30/93), 30.4\% (28/92), and 21.1\% (20/95)), leucopenia (21.5\% (20/93), $22.8 \%(21 / 92)$, and $15.8 \%(15 / 95))$, nausea (15.1\% (14/93), $16.3 \%(15 / 92)$, and $12.6 \%(12 / 95))$, and fatigue $(10.8 \%(10 / 93), 7.6 \%$ (7/92), and $8.4 \%(8 / 95))$ (Table 3).

\section{Discussion}

As neoadjuvant chemotherapy has been gradually accepted by clinical physicians, we found that the R0 resection is no longer difficult to achieve [9]. However, there are still quite a proportion of the patients who did not have survival benefits even with R0 resection. Compared with R0 resection, pathological remission may be a predictive parameter for survival [10-12]. Pathological regression might be a good short-term evaluation method for neoadjuvant chemotherapy for GC and a predictive parameter for survival [13].

Hence, the commonly used neoadjuvant chemotherapy regimens are difficult to meet the requirement of pathological remission. Based on the results of previous studies [8], we speculate that young Chinese patients with advanced GC with better physical conditions may improve the pathological remission rate with the treatment of a three-drug regimen of neoadjuvant chemotherapy. Therefore, the inclusion criteria of this trial were advanced GC patients under 60 years old and ECOG performance status of $0-1$.

Compared with the changes in TNM staging, RECIST, and tumor volume reduction rate, pathological regression is the most accurate factor to evaluate the efficacy of neoadjuvant chemotherapy, so the primary endpoint of this trial is pathological complete regression. The proportion of patients who achieved pCR with DOX, which was $16.1 \%$ in our study, is also inconsistent with previous docetaxel-based three-drug regimen reports [14-16]; the proportion of patients who achieved pathological complete regression in these studies ranged between $14 \%$ and $20 \%$. It is worth noting that the number of patients with complete pathological regression of DOX regimen was consistent with the response range of chemoradiation for gastroesophageal junction tumors and even exceeds that of chemoradiation [17]. For instance, the POET trial showed that $14.3 \%$ of patients with gastroesophageal junction adenocarcinoma achieved pathological complete regression with induction chemotherapy followed by chemoradiation [18]. According to the results of this study, neoadjuvant chemotherapy with either three- or two-drug regimens reduced the rate of lymph node metastasis but did not improve the rate of $\mathrm{R} 0$ resection.

In terms of nonsurgical adverse events, both DOX and XELOX were well tolerated and the incidences of adverse events were in line with previous reports in the perioperative setting. The main adverse reactions were leucopenia, neutropenia, and gastrointestinal symptoms [19-21].
One of the reasons for the low dropout rate in this study is that all the patients enrolled were confirmed without intraperitoneal implantation metastasis or negative for intraperitoneal abscission cytology in preoperative laparoscopic exploration. The aim is to reduce the shedding rate, which reflects the insufficiency of endoscopic and CT imaging staging and the importance of laparoscopic exploration before the treatment of GC [22]. At the same time, the DOX and XELOX groups performed laparoscopic exploration before neoadjuvant chemotherapy and before surgery again. Even so, 1 patient with positive peritoneal abscess cytology was found in each group, which indicated the importance of repeat laparoscopic exploration pre- and postneoadjuvant therapy [23].

Meanwhile, this study has some limitations. First, the selection of population was very different from real-life GC patients. The results of this study suggest that in the selection of patients for gastrectomy in metastatic esophagogastric cancer, clinical and pathological features are not enough; future prospective trials integrating tumor biology among inclusion criteria may help for defining the optimal candidates [24]. Second, pCR is an effective indicator of shortterm efficacy, and whether it can prolong the overall survival of patients or not is still controversial [13,17], which needs to be verified by further follow-up results. Third, we could not determine the optimal cycles of preoperative chemotherapy, as different cycles were not evaluated. Future trials will be needed to analyze the efficacy of different preoperative chemotherapy cycles.

In conclusion, the docetaxel-based triplet DOX significantly increased the proportion of patients achieving pathological complete regression compared with XELOX. At present, near half of the enrolled cases have benefited in terms of short-term efficacy. We will continue to recruit patients to expect more favorable results; the favorable pathological regression with DOX translates into better survival outcomes. DOX is expected to become one of the standard regimens for perioperative therapy of young and middleaged patients with advanced GC.

\section{Data Availability}

The [participant data with identifiers] data used to support the findings of this study were supplied by [Qun Zhao] under license and so cannot be made freely available. Requests for access to these data should be made to [Qun Zhao, zhaoqun@hebmu.edu.cn].

\section{Ethical Approval}

The present study was approved by the Ethical Review Committee of Hebei Medical University (Shijiazhuang, China).

\section{Consent}

Written informed consent was obtained from each patient. 


\section{Conflicts of Interest}

The authors have declared that no competing interests exist.

\section{Authors' Contributions}

Zhao Q designed the research; Li Y collected clinical data and performed research; Fan LQ, Yang PG, Zhang ZD, Zhao XF, Tan BB, and Wang D performed experiments; Tan BB and Tian Y analyzed data; and Zhao Q and Tian Y wrote the paper. All authors approved the final version of the manuscript.

\section{Acknowledgments}

This study was supported by the University research project of Hebei province (grant no. ZD2019139) and Medical research project of Hebei province (grant no. 20201137).

\section{References}

[1] D. Reim, M. Loos, F. Vogl et al., "Prognostic implications of the seventh edition of the International Union Against Cancer classification for patients with gastric cancer: the Western experience of patients treated in a single-center European institution," Journal of Clinical Oncology, vol. 31, no. 2, pp. 263-271, 2013.

[2] National Comprehensive Cancer Network, "(NCCN) Clinical Practice Guidelines in Oncology," Gastric Cancer, 2021, Version 2.

[3] J. Hoeppner, F. Lordick, T. Brunner et al., "ESOPEC: prospective randomized controlled multicenter phase III trial comparing perioperative chemotherapy (FLOT protocol) to neoadjuvant chemoradiation (CROSS protocol) in patients with adenocarcinoma of the esophagus (NCT02509286)," BMC Cancer, vol. 16, no. 1, p. 503, 2016.

[4] S. E. al-Batran, N. Homann, C. Pauligk et al., "Effect of neoadjuvant chemotherapy followed by surgical resection on survival in patients with limited metastatic gastric or gastroesophageal junction cancer," JAMA Oncology, vol. 3, no. 9, pp. 1237-1244, 2017.

[5] S. Lorenzen, A. Biederstädt, U. Ronellenfitsch et al., "RACEtrial: neoadjuvant radiochemotherapy versus chemotherapy for patients with locally advanced, potentially resectable adenocarcinoma of the gastroesophageal junction - a randomized phase III joint study of the AIO, ARO and DGAV," BMC Cancer, vol. 20, no. 1, article 7388, p. 886, 2020.

[6] N. Solomon, D. Mezentsev, I. Reis et al., "A phase II study of neoadjuvant and adjuvant chemotherapy with 5-fluorodeoxyuridine, leucovorin, oxaliplatin and docetaxel in the treatment of previously untreated advanced esophageal adenocarcinoma," Japanese Journal of Clinical Oncology, vol. 41, no. 4, pp. 469-476, 2011.

[7] Q. Zhao, Y. Li, J. Huang et al., "Short-term curative effect of S1 plus oxaliplatin as perioperative chemotherapy for locally advanced gastric cancer: a prospective comparison study," Pharmazie, vol. 72, no. 4, pp. 236-240, 2017.

[8] Q. Zhao, C. H. Lian, Z. B. Huo et al., "The efficacy and safety of neoadjuvant chemotherapy on patients with advanced gastric cancer: a multicenter randomized clinical trial," Cancer Medicine, vol. 9, no. 16, pp. 5731-5745, 2020.
[9] A. E. Slagter, E. P. M. Jansen, H. W. M. van Laarhoven et al., "CRITICS-II: a multicentre randomised phase II trial of neoadjuvant chemotherapy followed by surgery versus neoadjuvant chemotherapy and subsequent chemoradiotherapy followed by surgery versus neo-adjuvant chemoradiotherapy followed by surgery in resectable gastric cancer," BMC Cancer, vol. 18, no. 1, article 4770, p. 877, 2018.

[10] K. Becker, R. Langer, D. Reim et al., "Significance of histopathological tumor regression after neoadjuvant chemotherapy in gastric adenocarcinomas," Annals of Surgery, vol. 253, no. 5, pp. 934-939, 2011.

[11] S. Mukherjee, C. N. Hurt, S. Gwynne et al., "NEOSCOPE: a randomised phase II study of induction chemotherapy followed by either oxaliplatin/capecitabine or paclitaxel/carboplatin based chemoradiation as pre-operative regimen for resectable oesophageal adenocarcinoma," BMC Cancer, vol. 15 , no. 1 , p. $48,2015$.

[12] C. L. Donohoe, N. J. O'Farrell, T. Grant et al., "Classification of pathologic response to neoadjuvant therapy in esophageal and junctional cancer," Annals of Surgery, vol. 258, no. 5, pp. 784792, 2013.

[13] S. Lorenzen, P. Thuss-Patience, S. E. al-Batran et al., "Impact of pathologic complete response on disease-free survival in patients with esophagogastric adenocarcinoma receiving preoperative docetaxel-based chemotherapy," Annals of Oncology, vol. 24, no. 8, pp. 2068-2073, 2013.

[14] C. Schulz, F. Kullmann, V. Kunzmann et al., "NeoFLOT: multicenter phase II study of perioperative chemotherapy in resectable adenocarcinoma of the gastroesophageal junction or gastric adenocarcinoma-very good response predominantly in patients with intestinal type tumors," International Journal of Cancer, vol. 137, no. 3, pp. 678-685, 2015.

[15] P. C. Thuss-Patience, R. D. Hofheinz, D. Arnold et al., "Perioperative chemotherapy with docetaxel, cisplatin and capecitabine (DCX) in gastro-oesophageal adenocarcinoma: a phase II study of the Arbeitsgemeinschaft Internistische Onkologie $(\mathrm{AIO})^{\dagger}, "$ Annals of Oncology, vol. 23, no. 11, pp. 2827-2834, 2012.

[16] S. E. al-Batran, R. D. Hofheinz, C. Pauligk et al., "Histopathological regression after neoadjuvant docetaxel, oxaliplatin, fluorouracil, and leucovorin versus epirubicin, cisplatin, and fluorouracil or capecitabine in patients with resectable gastric or gastro-oesophageal junction adenocarcinoma (FLOT4AIO): results from the phase 2 part of a multicentre, openlabel, randomised phase 2/3 trial," The Lancet Oncology, vol. 17, no. 12, pp. 1697-1708, 2016.

[17] J. Y. An, H. I. Kim, J. H. Cheong, W. J. Hyung, C. B. Kim, and S. H. Noh, "Pathologic and oncologic outcomes in locally advanced gastric cancer with neoadjuvant chemotherapy or chemoradiotherapy," Yonsei Medical Journal, vol. 54, no. 4, pp. 888-894, 2013.

[18] M. Stahl, M. K. Walz, J. Riera-Knorrenschild et al., "Preoperative chemotherapy versus chemoradiotherapy in locally advanced adenocarcinomas of the oesophagogastric junction (POET): long-term results of a controlled randomised trial," European Journal of Cancer, vol. 81, pp. 183-190, 2017.

[19] K. Amarantidis, N. Xenidis, L. Chelis et al., "Docetaxel plus oxaliplatin in combination with capecitabine as first-line treatment for advanced gastric cancer," Oncology, vol. 80, no. 5-6, pp. 359-365, 2011

[20] K. R. Schønnemann, M. Yilmaz, M. Andersen, and P. Pfeiffer, "New recommendation of doses in an ongoing phase II study 
of docetaxel, oxaliplatin and capecitabine as first line therapy in advanced gastro-oesophageal cancer," Acta Oncologica, vol. 50, no. 1, pp. 151-152, 2011.

[21] S. J. Sym, M. H. Ryu, H. J. Kang et al., "Phase I study of 3weekly docetaxel, capecitabine and oxaliplatin combination chemotherapy in patients with previously untreated advanced gastric cancer," Cancer chemotherapy and pharmacology, vol. 66, no. 2, article 1171, pp. 373-380, 2010.

[22] D. Mahadevan, A. Sudirman, P. Kandasami, and G. Ramesh, "Laparoscopic staging in gastric cancer: an essential step in its management," Journal of minimal access surgery, vol. 6, no. 4, pp. 111-113, 2010.

[23] C. A. Thiels, N. Ikoma, K. Fournier et al., "Repeat staging laparoscopy for gastric cancer after preoperative therapy," Journal of surgical oncology, vol. 118, no. 1, pp. 61-67, 2018.

[24] L. Fornaro, V. Fanotto, G. Musettini et al., "Selecting patients for gastrectomy in metastatic esophago-gastric cancer: clinics and pathology are not enough," Future Oncology, vol. 13, no. 25, pp. 2265-2275, 2017. 